\title{
PROPAGACIÓN VEGETATIVA DE TORNILLO Cedrelinga cateniformis (Ducke) MEDIANTE ENRAIZAMIENTO DE ESTACAS JUVENILES EN PROPAGADOR DE SUBIRRIGACIÓN
}

\author{
Manuel SOUDRE ${ }^{1}$; Leisy MUERAS ${ }^{2}$; Aparicio LIMACHE ${ }^{2}$; Hector GUERRA ${ }^{1}$; Francisco MESEN ${ }^{3}$; \\ Fernando PEREZ ${ }^{2}$ \\ 1 Instituto de Investigaciones de la Amazonía Peruana (IIAP). Programa de investigación en Manejo Integral del Bosque y Servicios \\ Ambientales (PROBOSQUES).Ucayali, Perú. E-mails: msoudre@iiap.org.pe; torwhar@hotmail.com \\ 2 Universidad Nacional de Ucayali (UNU). Facultad de Ciencias Forestales. Ucayali, Perú \\ 3 Centro Agronómico Tropical de Investigación y Enseñanza (CATIE). Turrialba, Costa Rica. E-mail: fmesen@catie.ac.cr
}

\section{RESUMEN}

Se determinó el efecto de diferentes concentraciones de ácido indol-3-butírico (AIB), tipos de sustratos y las características vegetativas, sobre el enraizamiento de estacas juveniles (estaquillas) del tornillo (Cedrelinga cateniformis Ducke), mediante dos experimentos consecutivos en propagadores de subirrigación. En el primero se probó cinco dosis de AIB $(0,1000,2000,4000$ y $8000 \mathrm{ppm})$ y tres sustratos (arena fina, arena gruesa y grava fina), bajo un diseño de bloques completos al azar en parcelas divididas con cinco repeticiones. En el segundo experimento se probó tres tipos de estaquillas (apical, media y basal), dos áreas foliares $\left(15 \mathrm{y} 30 \mathrm{~cm}^{2}\right)$ y dos longitudes de estacas $(4$ y $8 \mathrm{~cm})$, usando un diseño de bloques completamente al azar con arreglo factorial $(3 \times 2 \times 2)$ con tres repeticiones; en este caso, las estaquillas fueron puestas a enraizar en el mejor sustrato (arena fina) y con la mejor concentración de AIB ( $4000 \mathrm{ppm}$ ) obtenidos en el primer ensayo. Se obtuvo un enraizamiento aceptable de $70 \%$, con longitud de raíz promedio $1.2 \mathrm{~cm}$ y número de raíces promedio de 4.6 por estaquilla utilizando estaquillas del tipo media, con área foliar de $30 \mathrm{~cm}^{2}, 4000 \mathrm{ppm}$ de AIB, en arena fina y en propagador de subirrigación.

PALABRAS CLAVE: Cedrelinga cateniformis, enraizamiento, estacas juveniles, sustratos, longitud de estaca y área foliar.

\section{VEGETATIVE PROPAGATION OF TORNILLO Cedrelinga cateniformis (Ducke) BY ROOTING OF JUVENILE CUTTINGS IN A NON-MIST PROPAGATOR}

\begin{abstract}
This study evaluated the effects of different concentrations of Indol-3-butiryc acid (IBA), rooting media and vegetative characteristics on the rooting of juvenile stem cuttings of tornillo (Cedrelinga cateniformis Ducke) in two consecutive experiments using non-mist propagators. In the first experiment, five IBA concentrations $(0,1000$, 2000,4000 and $8000 \mathrm{ppm}$ ) and three rooting media (fine sand, coarse sand and fine gravel) were tested, using a randomized complete block-split plot design with five replications. In the second experiment, three types of cutting (apical, middle and basal), two leaf areas $\left(15\right.$ and $\left.30 \mathrm{~cm}^{2}\right)$ and two cutting lengths $(4$ and $8 \mathrm{~cm})$ were evaluated, using a randomized complete block design with factorial arrangement $(3 \times 2 \times 2)$ and three replications. In this case, cuttings were set to root in the best rooting medium (fine sand) and with the best IBA concentration $(4000 \mathrm{ppm})$ as determined in the previous experiment. An acceptable rooting percentage of $70 \%$, an average root length of $1.2 \mathrm{~cm}$ and a mean number of roots of 4,6 per cutting were obtained using middle cuttings with leaf areas of $30 \mathrm{~cm}^{2}, 4000 \mathrm{ppm}$ of IBA and fine sand as rooting medium in the non-mist propagator.
\end{abstract}

KEYWORDS: Cedrelinga cateniformis, rooting, juvenile stem cuttings, rooting medium, cutting length, leaf area. 


\section{INTRODUCCIÓN}

Tornillo (Cedrelinga cateniformis Ducke), es un árbol nativo de la Amazonía que produce una de las maderas de mayor demanda en el mercado, desde hace casi cinco décadas (Aróstegui \& Díaz, 1992; Valderrama, 2003; Tello et al., 2004; Gonçalvez \& Gonçalvez, 2001; Ponce-Álvarez, 2005). Debido a su adecuada adaptación (Claussi et al., 1992) y buen crecimiento y productividad en plantaciones puras (Flores, 2010), es considerada una especie promisoria para la reforestación.

No obstante, es una especie extremadamente recalcitrante, debido a que sus semillas pierden totalmente su viabilidad a los pocos días de ser cosechadas (Schwyzer, 1981; Maruyama, 1987; Flores, 2004). Asimismo, presenta fructificación irregular, con producciones espaciadas, a veces, de hasta tres años (Maruyama, 1987). Adicionalmente, en el pasado tuvo una abundancia destacable que era favorecida por su regeneración natural agrupada denominada "tornillales"; sin embargo, actualmente es una especie que se encuentra en situación vulnerable (IUCN, 2007); incluso amenazada en algunos sectores amazónicos (P.A.P, 2001). Actualmente viene atravesando un severo proceso de erosión genética, es decir, la pérdida o deterioro de muchas características genéticas de alto valor, y la disminución de su capacidad reproductiva (Loayza, 2004). Por lo tanto, la escasa disponibilidad de árboles selectos y semillas en el corto plazo podría restringir severamente su existencia, e incluso la producción de plantones para la reforestación.

La propagación vegetativa es una alternativa prometedora para conservar la diversidad genética de germoplasma valioso y aumentar la ganancia genética en períodos muy cortos (Zobel \& Talbert, 1988). Pudiendo evitar la fuerte dependencia por semillas botánicas provenientes de rodales naturales de procedencia desconocida; incrementando las posibilidades de una oferta sostenible de semilla vegetativa durante todo el año y multiplicando los genotipos superiores de la especie que aún quedan. Cabe destacar, que durante la década de los años ochenta se realizaron varios esfuerzos para propagar vegetativamente al tornillo, usando para ello estacas leñosas, estimulantes hormonales y diversos microambientes en viveros, pero obtuvieron resultados muy poco alentadores $(0 \%)$, siendo calificada de muy difícil enraizamiento (Schwyzer, 1981; Castillo 1983; Maruyama, 1986; Claussi, 1986; Levi, 1987; Aróstegui, 1989).

Aunque una reciente experiencia obtuvo resultados alentadores (30\%), usando solo estaquillas foliadas, sustrato arena y en propagador de subirrigación (Soudre \& Portal, 2007). En consecuencia, es posible que el uso de material fisiológicamente juvenil, sustratos de buen drenaje y un control adecuando de las condiciones microambientales podrían influir en el éxito de su enraizamiento.

Por lo tanto, considerando los escasos resultados satisfactorios en su propagación vegetativa, el IIAP, con el apoyo financiero de FINCyT ejecutaron un proyecto, con el objetivo de determinar el porcentaje de enraizamiento de estaquillas de tornillo por efecto de cinco dosis de AIB (0, 1000, 2000, 4000 and 8000 $\mathrm{ppm}$ ), tres tipos de sustrato (arena fina, arena gruesa y grava fina) y tres características de estaquilla (tipo, longitud y área foliar), bajo condiciones de propagador de subirrigación.

\section{MATERIAL Y MÉTODOS}

\section{ÁREA EXPERIMENTAL}

Los experimentos se realizaron al interior del propagador de subirrigación que se instaló en el vivero forestal del Instituto de Investigación de la Amazonia Peruana (IIAP) en la región de Ucayali, Perú. Localizado a 539624 E, 9071577 N; altitud de 153 msnm, precipitación media anual de $1,943 \mathrm{~mm}$, humedad relativa promedio de $84.6 \%$, y temperatura media anual de $27^{\circ} \mathrm{C}$ (UNU, 2011).

El propagador de subirrigación (Figura 1) es una estructura muy simple, construida con listonería de madera de $2.5 \times 1.0 \times 1.0 \times 0.5 \mathrm{~m}$ de dimensión total, que luego fue forrada totalmente con polietileno transparente $\mathrm{N}^{\circ} 10$, según el diseño propuesto por Leakey et al. (1990) y Longman (1993). Tiene el propósito de albergar las estaquillas instaladas sobre los sustratos experimentales, bajo condiciones microambientales controladas, evitando así la pérdida de humedad y facilitando la entrada de luz hacia las estaquillas. Adicionalmente el propagador fue protegido de la radiación solar directa utilizando sombreamiento de $80 \%$ con malla Rashell de color negro, esta última se ubicó a dos metros desde la base del propagador. Las condiciones microambientales promedio al interior de este propagador fueron, temperatura $\left(29.1{ }^{\circ} \mathrm{C}\right)$, humedad relativa $(70.5 \%)$, temperatura de sustrato $\left(26.9{ }^{\circ} \mathrm{C}\right)$ e intensidad lumínica de 4,328 lux, respectivamente. Estos valores son coincidentes a lo recomendado por Botti (1999), es decir, no sobrepasaron los $30{ }^{\circ} \mathrm{C}$ y la humedad relativa fue mayor de $60 \%$, lo cual favoreció la hidratación de las estaquillas. Otras experiencias con especies tropicales confirman que la temperatura óptima del aire que favorecen al enraizamiento es de 20 a $25^{\circ} \mathrm{C}$, aunque temperaturas hasta $30{ }^{\circ} \mathrm{C}$ son aceptables siempre y cuando se mantenga una humedad relativa muy alta (Leakey \& Mesén, 1991). 

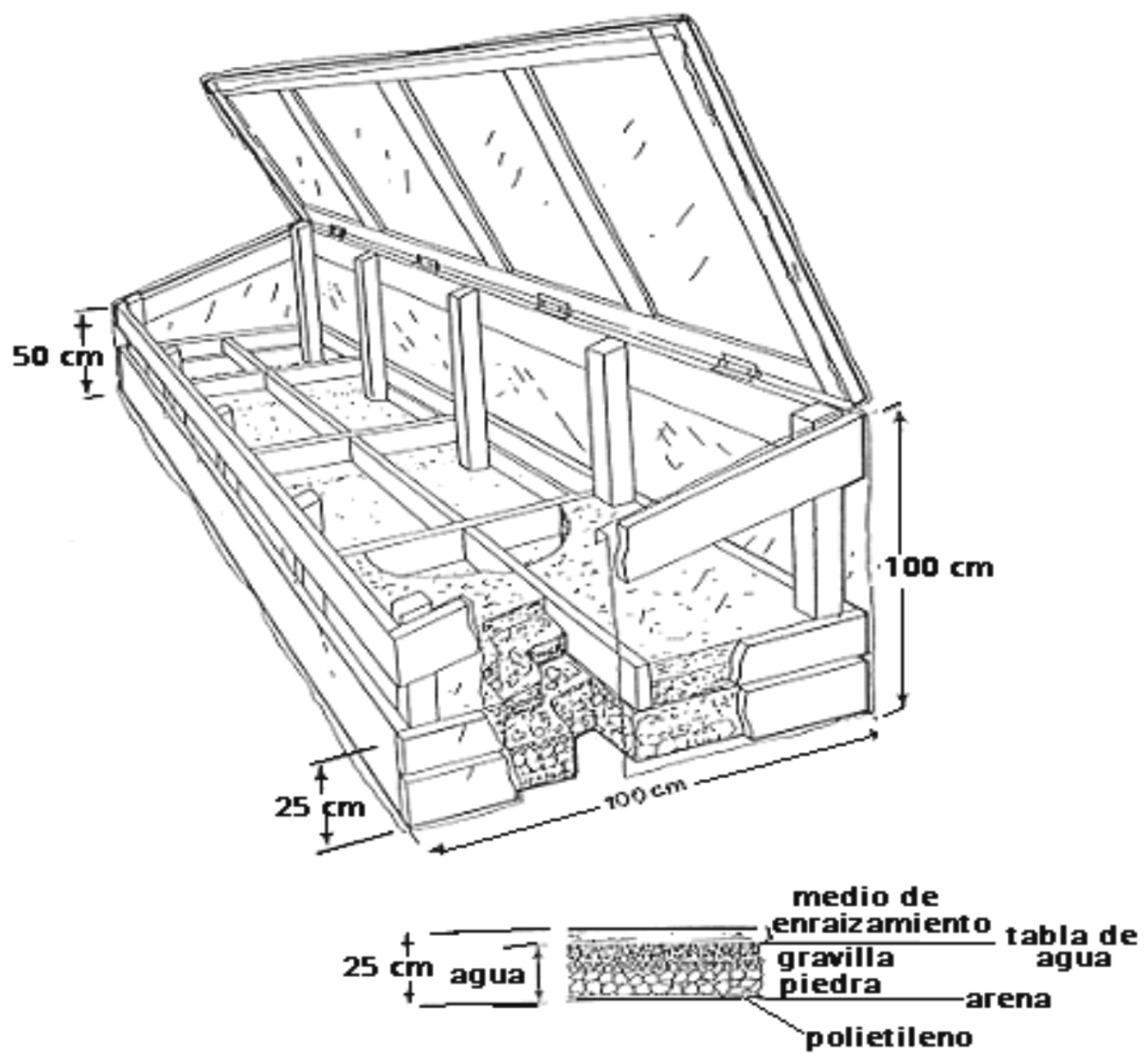

Figura 1. Características del propagador de subirrigación (Leakey et al., 1990; Longman, 1993).

\section{METODOLOGÍA}

Las estaquillas de tornillo se obtuvieron de los segmentos del tallo principal a partir de plantones originados de semillas, de siete meses de edad, producidos y manejados en el mismo vivero forestal. Las semillas empleadas procedieron de un árbol selecto de la plantación P401-IIAP, ubicada en la estación experimental Jenaro Herrera, región Loreto.

Los plantones fueron cortados y dimensionados en pequeños segmentos denominados estaquillas, definidos por los entrenudos. La preparación de estaquillas del segundo experimento, siguió el mismo procedimiento del primer experimento, pero se tuvo en cuenta una longitud específica y una superficie equivalente al área foliar requerida para cada tratamiento. Todas las estaquillas fueron desinfectadas durante 10 a 15 minutos en solución fúngica de oxicloruro de cobre (Cupravit) al $0.3 \%$ y luego oreadas por 10 minutos más. La solución de AIB fue preparada disolviendo una cantidad específica de auxina en alcohol puro $\left(96^{\circ}\right)$, con el fin de obtener la concentración deseada. La solución hormonal fue aplicada en la base de la estaquillas durante tres a cinco segundos y luego se provocó la evaporación inmediata del alcohol a través de una corriente de aire generada por un ventilador, durante 20 segundos más. Finalmente, todas las estaquillas fueron instaladas en los sustratos experimentales al interior de propagador.

Los sustratos fueron previamente lavados, esterilizados con vapor de agua, secados por solarización y finalmente clasificados según su granulometría (grosor de partículas), para el caso de arena fina de 0.1 a $2 \mathrm{~mm}$ de diámetro, se usó el tamiz $\mathrm{N}^{\circ} 40$; para arena gruesa 1 a $2 \mathrm{~mm}$, el tamiz $\mathrm{N}^{\circ} 20$; y para la grava fina 2 a $5 \mathrm{~mm}$, el tamiz $\mathrm{N}^{\circ} 12$. Las estaquillas fueron insertadas en pequeños hoyos de 2 $\mathrm{cm}$ de profundidad y a $5 \times 5 \mathrm{~cm}$ de distanciamiento entre hoyos, previamente elaborados en cada sustrato. El manejo y el control microambiental del propagador de subirrigación, fue el mismo para ambos ensayos. También se realizó el monitoreo semanal del desarrollo radicular, a fin de determinar el momento oportuno de repique y la fecha de finalización de los ensayos. 


\section{DISEÑO EXPERIMENTAL}

En el primer experimento, se probaron cinco dosis de AIB $(0,1000,2000,4000$ y 8000 ppm) y también tres tipos de sustratos (arena fina, arena gruesa y grava fina). Se utilizó un diseño bloques completamente al azar (DBCA) en parcelas divididas, con cinco repeticiones, quince tratamientos y seis estaquillas por unidad experimental; donde las parcelas grandes fueron los sustratos y las parcelas pequeñas a las diferentes dosis de AIB. Las estaquillas tuvieron una longitud promedio de $6 \mathrm{~cm}$ y una hoja recortada a la mitad $\left(15 \mathrm{~cm}^{2}\right)$. El experimento tuvo una duración de 50 días y se utilizó un total de 450 estaquillas.

El segundo experimento, fue establecido una vez concluido el primero, con el propósito de identificar el mejor sustrato (arena fina) y la mejor dosis de AIB (4000 ppm) resultantes. En este experimento se evaluó el efecto de tres tipos de estaquillas (apical, media y basal), dos longitudes de estacas (4 y $8 \mathrm{~cm}$ ) y dos áreas foliares $\left(15\right.$ y $30 \mathrm{~cm}^{2}$ ), para lo cual se utilizó el diseño de bloques completamente al azar con arreglo factorial $3 \times 2 \times 2$, con 12 tratamientos, tres repeticiones y diez estaquillas por cada unidad experimental. Se requirió un total de 360 estaquillas y la experimentación se realizó en 40 días.

\section{PROCESAMIENTO DE DATOS}

Se generó una base de datos en el software Microsoft Excel, luego determinó la capacidad de enraizamiento de las estaquillas, en términos del: a) porcentaje de enraizamiento, b) longitud de raíces y c) número de raíces; los valores promedios de estas variables por cada unidad experimental fueron analizadas mediante el procedimiento de Análisis de Varianza (ANOVA) y luego sometidos a la prueba de comparación de medias de Tukey $(p<0.05)$ para determinar la naturaleza de las diferencias entre tratamientos. Previamente las variables expresadas en porcentaje fueron transformadas con la función arcsen $\sqrt{\%}$; pero posteriormente, para su interpretación, los valores promedios fueron convertidos a las unidades originales. Se utilizó el software estadístico InfoStat.

\section{RESULTADOS Y DISCUSIÓN}

\section{EFECTO DEL TIPO DE SUSTRATO}

Siete semanas después de instalar el primer experimento, se determinó la influencia significativa $(p<0.05)$ de la interacción tipo de sustrato*dosis de $A I B$ sobre el porcentaje de enraizamiento de estaquillas de tornillo, es decir, que el sustrato no habría actuado independientemente, sino que el enraizamiento estaría influenciado por la acción combinada entre el tipo de sustrato y la dosis de AIB, a su vez. Cabe destacar, que cuando el sustrato fue analizado independientemente el porcentaje de enraizamiento no logró superar los $52.7 \%$ de enraizamiento, pero en respuesta a la influencia combinada de ambos factores a la vez (arena fina y 4000 ppm de AIB) se logró un 64\% de enraizamiento, la cual fue estadísticamente similar $(p<0.05)$ a la dosis de AIB más alta (8000 ppm).

Aunque la dependencia generada por el efecto combinado de la interacción no permite identificar con precisión el nivel de influencia de cada factor, sí fue posible evidenciar la superioridad del impacto del tipo de sustrato frente a la dosis de AIB, dado que el porcentaje de enraizamiento de estaquillas sin dosis hormonal (testigo $=0$ ppm) también tuvo un crecimiento significativo, desde los sustratos más gruesos hacia los más finos, en grava $(7 \%)$, arena gruesa $(27 \%)$, y con arena fina $(53 \%)$, incluso, este último, estuvo muy cerca del tratamiento más exitoso de todo el experimento. Frente a la evidencia, donde las estaquillas de tornillo sin hormona también enraízan hasta en el sustrato menos adecuado (7\%), se confirmaría que el enraizamiento de tornillo fue influenciado de forma sobresaliente por el tipo de sustrato, más que la propia hormona (Tabla 1).

Es posible que el tipo de arena esté proporcionando la aireación, drenaje y soporte apropiados para que las estaquillas de tornillo enraícen relativamente mucho más (Wright, 1964; Hartmann \& Kester, 1972; Davidson, 1974; Poggiani \& Suiter Filho, 1976). El resultado concuerda con lo expresado por Quijada (1980), quien afirma que un sustrato debe garantizar una humedad sin excesos y esto se logra con una textura adecuada. La arena como medio de enraizamiento dio buenos resultados en Gmelina arborea y muchas especies forestales (Mesén, 1992). Debido posiblemente al buen balance entre aireación y humedad de las partículas de arena en comparación con la grava (Mesén, 1998). Además, está demostrado que la arena es un sustrato relativamente económico, disponible y fácil de esterilizar.

No se descarta que las estaquillas de tornillo puedan haber tenido en su estructura una cantidad mínima de sustancias estimuladoras para el enraizamiento, considerando que el material es fisiológicamente juvenil. Este último evento podría ser atribuido al contenido endógeno de auxinas que presentan las estacas en el momento de ser propagadas (Hartmann \& Kester, 1998). Aunque definitivamente la adición dosis intermedias en la base de la estaquillas fue la cantidad más apropiada para fomentar el desarrollo inicial de raíces adventicias. Este hallazgo también fue corroborado por quienes usaron dosis intermedias (2000 a 4000 ppm) de AIB en sustratos arenosos para las especies Cedrela odorata; Swietenia macrophylla; 
Eucalyptus deglupta; Bombacopsis quinata; Platymiscium pinnatum; G. arbórea; Alnus acuminata (Leakey et al., 1990; Díaz et al., 1991; Mesén et al., 1992 y 1996; Mesén, 1993; Mesén \& Trejos, 1997; Núñez, 1997).

\section{EFECTO DE LA DOSIS DE AIB}

Por su parte, la dosis de AIB influyó significativamente $(p<0.05)$ en la longitud de raíz promedio por estaquillas de tornillo. Destacando dos grupos de dosificación hormonal $(p<0.05)$, en el primer grupo, fue evidente el efecto favorable de las dosis bajas a intermedias de AIB (1000 a 4000 ppm) para un mayor alargamiento de las raíces $(0.98$ a 1.17 $\mathrm{cm}$ ) y de otra parte el grupo conformado por dosis extremas (testigo y $8000 \mathrm{ppm}$ ) obtuvieron en promedio raíces $41 \%$ más cortas $(0.61$ a $0.67 \mathrm{~cm})$. La prueba Tuckey $(p<0.05)$ permitió establecer que fueron las dosis de AIB (1000, 2000 y 4000 ppm) las que lograron estadísticamente las mayores longitudes promedio de raíces $(1.20,1.10$ y $0.98 \mathrm{~cm}$, respectivamente) a los 50 días; en contraste, tanto la dosis máxima (8000 ppm), como la dosis control (0 ppm) se mostraron adversas para estimular el alargamiento de la raíz. Los beneficios de la aplicación de auxinas en la división y alargamiento celular han sido demostrados ampliamente, favorecen el transporte de carbohidratos y co-factores hacia la base de la estaca, promoviendo así la iniciación y el desarrollo de las raíces (Haissig, 1974, citado por Núñez, 1997). Por lo tanto, las dosis bajas a intermedias de AIB son lo suficientes para obtener raíces más largas.

Las dosis de AIB también influyeron significativamente $(p<0.05)$ sobre el numero de raíces por estaquilla, mostrando una gradiente positiva entre ambas, desde 0 hasta $4000 \mathrm{ppm}$; contrariamente solo con la dosis extrema (8000 ppm) el número de raíces por estaca disminuyo, e incluso fue similar estadísticamente a la dosis de 2000 ppm. En Vochysia guatemalensis también el número de raíces producidas en las estacas aumentó con dosis crecientes de AIB desde 0 hasta $0.8 \%$ (Mesén \& Trejos, 1997). El objetivo de tratar con sustancias reguladoras (hormonas) es aumentar el porcentaje de estaquillas que formen raíces, acelerar su iniciación, aumentar la calidad, uniformidad y número de raíces por estaquilla (Hartmann \& Kester, 1998). Con la dosis intermedia de 4000 ppm de AIB, se logró obtener el máximo número de raíces (3.4 raíces/estaquilla), en contraste a las estaca que no tuvieron AIB (0 ppm) produjeron el menor número de raíces $(0.96$ raíces/estaquilla). Leakey (1985), menciona que es deseable que las estaquillas tengan muchas raíces, pero tres raíces bien ramificadas y distribuidas alrededor de las estacas son suficientes. Por lo tanto, usar una dosis intermedia de AIB (4000 ppm) es suficiente para obtener el mayor número de raíces en estaquillas de tornillo. El número de raíces promedio general por estaquilla fue de 2.2 unidades.

\section{EFECTO DEL TIPO DE ESTAQUILLA}

En el segundo experimento, el análisis de varianza detectó que el factor principal tipo de estaquilla influyó significativamente $(p<0.05)$ en el porcentaje de enraizamiento del tornillo (Tabla 2). La Figura 2 muestra la variación significativa en el porcentaje de enraizamiento debido a tres tipos de estaquillas, donde las del tipo medio obtuvieron el máximo enraizamiento registrado en ambos experimento $(70 \%)$. Este valor de enraizamiento se encuentra dentro del rango de clasificación aceptable (70 - 90\%). Incluso, para un programa operativo de clonación (Leakey, 1987). Cabe destacar, lo destacable de este resultado considerando que otros estudios realizados en el pasado, donde fue calificada como especie de muy difícil enraizamiento (Schwyzer, 1981; Castillo, 1983; Maruyama, 1986; Claussi, 1986; Levi, 1987; Aróstegui, 1989). Y mayor a los observados en otras especies con el mismo propagador de subirrigación, como G. arborea (Ruiz et al., 2005) y las coníferas Cupressus macrocarpa, Cupressus serpenvirens, Thuja orientalis y Chamaecyparis lawsoniana (Mateo etal., 2000).

Las estaquillas de tornillo presentaron la tendencia más común de enraizamiento, dado que son las estaquillas del tipo medio y basal las más promisorias para el enraizamiento, frente a las apicales (Brousse \& Loussert, 1980). Las estaquillas básales muestran mejor calidad de raíces y mayor cantidad de reservas que las estacas apicales (Soudre et al., 2008). Esto también fue confirmado por Hartmann et al., 1992, quien agrega que es debido a que en la composición química de las ramas hay marcadas diferencias desde la base a la punta. Así como, gradientes en suculencia, área foliar, edad, morfología y diferencial en el potencial de agua (Leakey, 1983). Solo en algunas especies, las estaquillas apicales muestran buen enraizamiento, esto ha sido atribuido a la mayor presencia de sustancias promotoras del enraizamiento (originadas en el ápice) y a una disímil calidad anatómica de la estaquilla apical para algunas especies (Mesén, 1998).

Además, el tipo de estaquilla también influyó significativamente $(p<0.05)$ en la longitud de raíz promedio (Tabla 2). Obteniendo $0.85 \mathrm{~cm}$ de longitud promedio, similar al del primer ensayo $(0.9 \mathrm{~cm})$, solo que en este caso el coeficiente de variabilidad fue mejor porque disminuyo en casi $20 \%(\mathrm{C} . \mathrm{V}=39.1 \%$ ), es 
decir, el tipo de estaquilla influiría en la mejor homogeneidad del desarrollo longitudinal de las raíces de cada estaquilla. Las estaquillas medias también presentaron las mayores longitudes de raíz $(1.01 \mathrm{~cm})$, seguidas por las basales $(0.88 \mathrm{~cm})$ y finalmente por estaquillas apicales $(0.66 \mathrm{~cm})$. Por lo tanto, las estaquillas cercanas a la base (media y basal) favorecen el mayor alargamiento de la raíz; en contraste, las estaquillas apicales mostraron en promedio raíces $31 \%$ más cortas.

\section{EFECTO DE LA LONGITUD DE ESTAQUILLA}

Se determinó la influencia significativa $(p<0.05)$ de la interacción longitud de estaquilla*tipo de estaquilla sobre el numero raíces promedio en estaquillas de tornillo (Tabla 3). Manteniendo fijos cada uno de los tres tipos de estaquillas (apical, media y basal), las estaquillas de posición media de 4 y $8 \mathrm{~cm}$ presentaron el mejor comportamiento lográndose el mayor número de raíces por estaquilla (4.5 y 4.6 unidades, respectivamente). Lo propio sucedió en un estudio con estaquillas de tipo media en la especie Plukenetia volubilis de $8 \mathrm{~cm}$ de longitud, obteniendo un valor máximo de 19 raíces por estaquilla (Ruiz, 2008). Por su parte, estaquillas de tornillo del tipo medio, tanto de 4 y $8 \mathrm{~cm}$, no tuvieron diferencias con las estaquillas apicales de $8 \mathrm{~cm}$ (4.4 raíces). En este último caso, al parecer un $100 \%$ más longitud adicional en las estaquillas apicales habría compensado las deficiencias anatómicas, traduciéndose en la cantidad de reservas de carbohidratos disponibles, considerando que las estaquillas apicales de $4 \mathrm{~cm}$ obtuvieron el menor número de raíces del ensayo (1.9 unidades). El número de raíces producido por las estaquillas es altamente influenciado por la habilidad de la estaquilla de suplir carbohidratos, ya sea de reserva o producidos mediante fotosíntesis, al área donde surgen las raíces (Moe \& Anderson, 1988; Veirskov \& Anderson, 1982, citado por Mesén, 1998). Por lo tanto, en el caso de tornillo quedaría demostrado que para fomentar el mayor número de raíces a los 40 días es posible usar, estaquillas medias, tanto de $4 \mathrm{~cm}$, como de $8 \mathrm{~cm}$, indistintamente.

\section{EFECTO DEL ÁREA FOLIAR DE LA ESTAQUILLA}

En cuanto al factor área foliar, se demostró su influencia significativa $(p<0.05)$ sobre el porcentaje de enraizamiento de estaquillas de tornillo. Estaquillas con $30 \mathrm{~cm}^{2}$ de área foliar obtuvieron el máximo enraizamiento (68\%) frente a las estaquillas de $15 \mathrm{~cm}^{2}$ de área foliar (52\%). El área foliar cercana a $50 \mathrm{~cm}^{2}$ produjo siempre los mejores resultados en el enraizamiento de muchas especies (Leakey \& Mesén, 1991). Así como un grupo importante especies forestales con áreas foliares intermedias, cercanas a 50 $\mathrm{cm}^{2}$ (Mesén, 1998).

La estaca debe conservar parte de la hoja, por ser esta fuente de asimilados, auxinas y otras sustancias, vitales para la capacidad de enraizamiento (Mesén, 1998). La presencia de hoja está relacionada con la producción de carbohidratos derivados de la fotosíntesis producción de promotores auxínicos, auxinas sinergistas (co-factores) o de nutrientes (Kamaluddin, 1996, citados por Núñez, 1997). Los promotores pueden ser transportados desde la hoja hasta la zona de enraizamiento en la base de la estaquilla, puesto que las hojas maduras exportan principalmente en una dirección basipétala (Wilson, 1994 citado por Núñez, 1997). Pese a ello, la hoja también proporciona una amplia superficie para la pérdida de agua por transpiración, por estas razones las hojas deben recortarse a un tamaño tal que se logre el mejor balance entre las desventajas de la transpiración y la ventaja de la fotosíntesis (Mesén, 1998). Una estaquilla sin hojas no podría arraigar y una estaca que pierde sus hojas en el transcurso del arraigue está igualmente condenada, pues aunque esté empezando a echar raíces, no podrá desarrollarse, por lo tanto, es necesario una superficie foliar mínima para asegurar la fotosíntesis suficiente para satisfacer las necesidades correspondientes al desarrollo del sistema radical y a la vida futura de estaca (Braudeau, 1981). El área foliar definitiva aparentemente resultaría de la necesidad de optimizar el balance entre los beneficios de la fotosíntesis y las desventajas de la transpiración (Leakey \& Mesén, 1991; Aminah et al., 1997). Esto varía entre las especies y clones, en función de niveles específicos de área foliar, espesor de hojas, la densidad estomática, la morfología de la hoja y la edad de la hoja (posición de nudo) (Leakey, 1985).

El máximo porcentaje de enraizamiento (68\%) fue logrado con $30 \mathrm{~cm}^{2}$ de área foliar, siendo muy similar al obtenido en el primer experimento con estaquillas del tipo medio (70\%), es decir, cuantitativamente el factor tipo de estaquilla presentaría un grado de importancia aproximadamente similar al factor de área foliar. Por tanto, existen evidencias suficientes para demostrar que la presencia de hojas es fundamental para el proceso de rizogenesis de esta especie, en consecuencia el ideótipo de tornillo más apropiado para obtener el máximo enraizamiento estaría compuesto por estaquillas tipo medio y de superficie foliar de $30 \mathrm{~cm}^{2}$.

De igual modo, el área foliar también influyó de forma altamente significativas $(p<0.05)$ en la longitud de raíz promedio por estaquilla. De hecho, la prueba de Tuckey $(p<0.05)$ para las dos áreas foliares determinó respuestas muy contrastantes para cada nivel de área foliar, encontrando que con $30 \mathrm{~cm}^{2}$ de área foliar se 
produce la máxima longitud de raíz promedio por estaquilla $(1.09 \mathrm{~cm})$, frente a los $0.62 \mathrm{~cm}$ obtenidos por las estaquillas con área foliar de $15 \mathrm{~cm}^{2}$ (Figura 3). En consecuencia, una vez más se confirma que para obtener raíces más largas, en al menos 40 días, también sería necesario utilizar estaquillas del tipo medio y $30 \mathrm{~cm}^{2}$ de área foliar.

Finalmente, el área foliar también influyó de forma altamente significativas $(p<0.05)$ en el número de raíces por estaquilla de tornillo. Con área foliar de 30 $\mathrm{cm}^{2}$ se produjo el mayor número de raíces por estaquilla (4.6 unidades), frente a 2.6 raíces obtenidas con estaquillas con área foliar de $15 \mathrm{~cm}^{2}$ (Figura 3). De este modo, también se corroboró que para obtener el máximo número de raíces se requiere el uso de estaquillas de tipo media y $30 \mathrm{~cm}^{2}$ de área foliar.

Tabla 1. Prueba de Tukey $(p<0.05)$ para el porcentaje de enraizamiento promedio de estaquillas de tornillo $C$. cateniformis por el efecto de la interacción de los factores tipo de sustrato ${ }^{*}$ dosis $A I B$, a los 50 días en propagador de subirrigación (primer experimento).

\begin{tabular}{lccc}
\hline $\begin{array}{c}\text { SUSTRATO } \\
\text { (TIP0) }\end{array}$ & $\begin{array}{c}\text { DOSIS AIB } \\
(\mathbf{p p m})\end{array}$ & $\begin{array}{c}\text { ENRAIZAMIENTO } \\
(\%)\end{array}$ & SIGNIFICANCIA \\
\hline Arena fina & 4000 & 64 & $\mathrm{~A}$ \\
\hline Arena fina & 8000 & 60 & $\mathrm{~A}, \mathrm{~B}$ \\
\hline Arena gruesa & 2000 & 57 & $\mathrm{~B}$ \\
\hline Arena fina & 0 & 53 & $\mathrm{~B}, \mathrm{C}$ \\
Arena gruesa & 1000 & 50 & $\mathrm{~B}, \mathrm{C}$ \\
Arena fina & 1000 & 50 & $\mathrm{~B}, \mathrm{C}$ \\
Grava fina & 4000 & 50 & $\mathrm{~B}, \mathrm{C}$ \\
Grava fina & 1000 & 43 & $\mathrm{~B}, \mathrm{C}, \mathrm{D}$ \\
\hline Arena fina & 2000 & 40 & $\mathrm{C}, \mathrm{D}, \mathrm{E}$ \\
\hline Arena gruesa & 4000 & 37 & $\mathrm{C}, \mathrm{D}, \mathrm{E}, \mathrm{F}$ \\
Arena gruesa & 0 & 27 & $\mathrm{D}, \mathrm{E}, \mathrm{F}$ \\
Grava fina & 2000 & 27 & $\mathrm{E}, \mathrm{F}, \mathrm{G}$ \\
Grava fina & 8000 & 23 & $\mathrm{~F}, \mathrm{G}$ \\
\hline Arena gruesa & 8000 & 17 & $\mathrm{G}$ \\
Grava fina & $0 \mathrm{~m}$ & 7 & $\mathrm{H}$ \\
\hline
\end{tabular}

$(A, B, C, D, E, F, G, H)$ Letras distintas indican diferencias significativas $(p<0.05)$

$\overline{\text { Tabla 2. Prueba de Tuckey }(p<0.05)}$ para los promedios del porcentaje del enraizamiento, longitud raíz y numero raíces, por efecto de tres tipos de estaquillas de tornillo $C$. cateniformis, a los 40 días en propagador de subirrigación (segundo experimento).

\begin{tabular}{cccc}
\hline \multirow{2}{*}{$\begin{array}{c}\text { ESTAQUILLA } \\
\text { (TIPO) }\end{array}$} & $\begin{array}{c}\text { ENRAIZAMIENTO } \\
(\%)\end{array}$ & $\begin{array}{c}\text { LONGITUD RAÍCES } \\
(\mathbf{c m})\end{array}$ & $\begin{array}{c}\text { NÚMERO RAÍcES } \\
\left(\mathbf{N}^{\mathbf{0}}\right)\end{array}$ \\
\cline { 2 - 4 } & $70 \boldsymbol{a}$ & $1.01 \boldsymbol{a}$ & $4.55 \boldsymbol{a}$ \\
Media & $62 \boldsymbol{b}$ & $0.88 \boldsymbol{b}$ & $2.83 \boldsymbol{b}$ \\
Basal & $48 \boldsymbol{c}$ & $0.66 \boldsymbol{c}$ & $3.14 \boldsymbol{b}$ \\
\hline Apical & & &
\end{tabular}

$(a, b, y c)$ Letras distintas indican diferencias significativas $(p<0.05)$ 
FOLIA

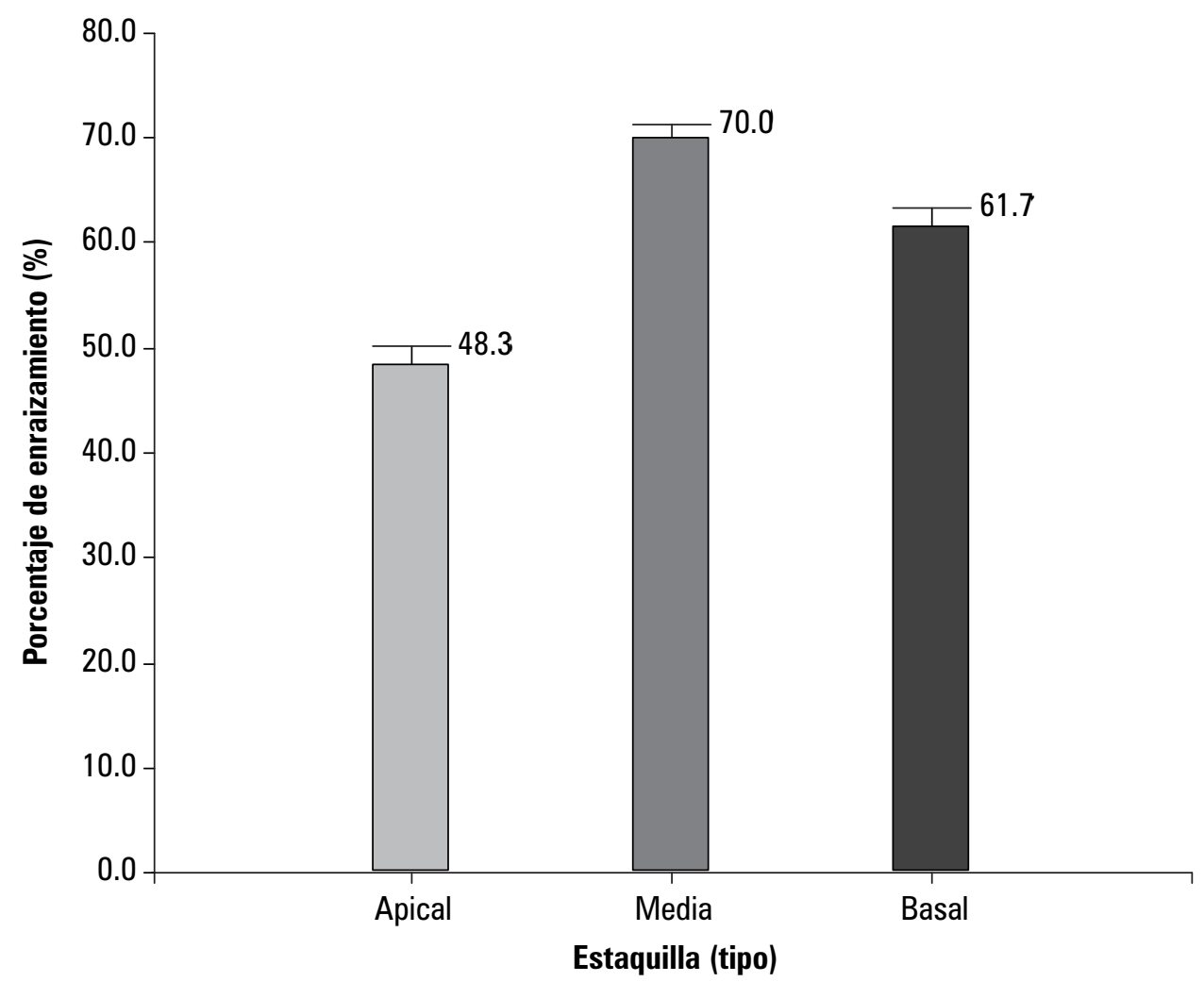

Figura 2. Efecto de tipo de estaquillas sobre el porcentaje de enraizamiento promedio de tornillo $C$. cateniformis, a los 40 días de instalación en propagador de subirrigación (segundo experimento).

Tabla 3. Prueba de Tukey $(\mathrm{p}<0.05)$ para el número de raíces promedio, por efecto de la interacción de los factores tipo de estaquilla*longitud de estaquilla de tornillo $C$. cateniformis, a los 40 días en propagador de subirrigación (segundo experimento).

\begin{tabular}{cccc}
\hline $\begin{array}{c}\text { ESTAQUILLA } \\
\text { (TIP0) }\end{array}$ & $\begin{array}{c}\text { LONGITUD DE ESTAQUILLA } \\
(\mathbf{c m})\end{array}$ & $\begin{array}{c}\text { NUMERO RAÍ́CES } \\
\left(\mathbf{N}^{\circ}\right)\end{array}$ & SIGNIFICANCIA \\
\hline Media & 4 & 4.6 & $\mathrm{~A}$ \\
Media & 8 & 4.4 & $\mathrm{~A}$ \\
Apical & 8 & 4.3 & $\mathrm{~A}$ \\
Basal & 4 & 2.9 & $\mathrm{~A}, \mathrm{~B}$ \\
Basal & 8 & 2.8 & $\mathrm{~A}, \mathrm{~B}$ \\
Apical & 4 & 1.9 & $\mathrm{~B}$ \\
\hline
\end{tabular}

$(A, B)$ Letras distintas indican diferencias significativas $(p<0.05)$ 
$\overline{\text { Tabla 4. Análisis de Varianza (ANOVA) }}$ para el porcentaje de enraizamiento de estaquillas de tornillo C. cateniformis, a los 40 días en propagador de subirrigación (segundo experimento).

\begin{tabular}{|c|c|c|c|c|c|}
\hline F.V. & SC & GL & CM & $\mathbf{F}$ & SIGNIFICANCIA \\
\hline Bloque & 0.01 & 2 & 0.01 & 18.64 & 0.0094 \\
\hline Tipo de estaquilla & 0.42 & 2 & 0.21 & 3.96 & $0.0339 *$ \\
\hline Longitud de estaquilla & 0.05 & 1 & 0.05 & 0.91 & 0.3495 \\
\hline Área foliar & 0.40 & 1 & 0.40 & 7.57 & $0.0116 *$ \\
\hline Tipo de estaquilla*Longitud de estaquilla & 0.10 & 2 & 0.05 & 0.96 & 0.3986 \\
\hline Tipo de estaquilla*Area foliar & $1.7 \mathrm{E}^{-03}$ & 2 & $8.5 \mathrm{E}^{-04}$ & 0.02 & 0.9840 \\
\hline Longitud de estaquilla*área foliar & 0.01 & 1 & 0.01 & 0.21 & 0.6476 \\
\hline$T$ estaquilla ${ }^{*} L$ estaquilla ${ }^{*} A$ foliar & 0.13 & 2 & 0.07 & 1.23 & 0.3108 \\
\hline Error experimental & 1.17 & 22 & 0.05 & & \\
\hline Total & & 35 & & & \\
\hline
\end{tabular}

Enraizamiento de estacas juveniles de Tornillo (Cedrelinga cateniformis) 40 días.

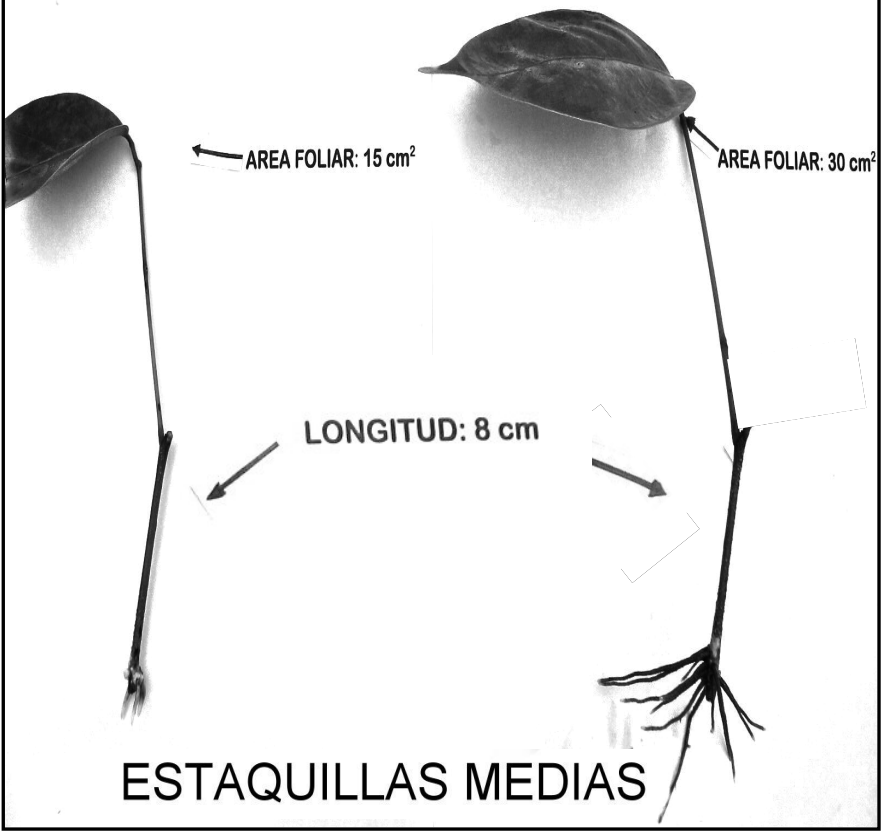

Figura 3. Comparativo entre los sistemas radiculares de estaquillas de tornillo $C$. cateniformis cuando estas presentan hojas de 15 y $30 \mathrm{~cm}^{2}$ de área foliar, a los 40 días en propagador de subirrigación (segundo experimento). 


\section{CONCLUSIONES}

Se comprobó que las estaquillas de tornillo $(C$. cateniformis) del tipo media, desde $4 \mathrm{~cm}$ de longitud, con área foliar de $30 \mathrm{~cm}^{2}, 4000 \mathrm{ppm}$ de AIB, puestas a enraizar en arena fina $(0.1-0.2 \mathrm{~mm})$ y bajo condiciones microambientales del propagador de subirrigación, obtuvieron a los 40 días el máximo porcentaje de enraizamiento de $70 \%$, longitud de raíz promedio de $1.2 \mathrm{~cm}$ y número de raíces promedio de 4.6 unidades por estaquilla. Se debe mejorar la técnica utilizando estaquillas juveniles provenientes de rebrotes basales de árboles adultos, previamente promover la producción y manejo de rebrotes bajo diversos niveles de luz, riego, fertilidad, tipo de corte, época de corte, uso de cicatrizante y podas de selección. No usar ramas leñosas de árboles, ya que su constitución fisiológica es contraria a la de una estaquilla.

\section{AGRADECIMIENTOS}

Al Fondo para la Innovación Ciencia y Tecnología (FINCyT) por el decidido apoyo financiero, a través del proyecto "Desarrollo tecnológico apropiado para la propagación vegetativa de especies maderables PROVEFOR- (Contrato $\mathrm{N}^{\circ}$ 013-PIBAP-FINCyTIIAP-2007), ejecutado por el IIAP. Con especial gratitud al equipo técnico y logístico liderado por el técnico Rony Ríos y el apoyo de Wilson Guerra, Floriel Flores, Marcos Flores y Joel Saboya.

\section{BIBLIOGRAFIA CITADA}

Aminah, H.; Dick, J.; Grace, J. 1997. Rooting of Shorea leprosula stem cuttings decreases with increasing leaf area. Forestal Ecology and Management(Holanda). 91:247-254.

Aróstegui, A. 1989. Propagación por estacas del tornillo (Cedrelinga catenaeformis Ducke) y goma pashaco (Parkia igneiflora Ducke). Instituto de Investigaciones de la Amazonía Peruana, Iquitos, Perú. $9 \mathrm{p}$

Aróstegui, A; Díaz, M. 1992. Propagación de especies forestales nativas promisorias en Jenaro Herrera. Instituto de Investigación de la Amazonia PeruanaIIAP. Iquitos, Perú. $119 \mathrm{p}$

Botti, C. 1999. Principios de la Propagación y Técnica de Propagación por estacas. En: Manejo Tecnificado de Invernadero y Propagación de Plantas. Departamento de Producción Agrícola. Facultad de Ciencias Agronómicas. Universidad de Chile. Santiago, Chile. P. 49-55.

Braudeau, J. 1981. El Cacao. Técnicas Agrícolas y Producciones Tropicales. Blume Distribuidora S.
A. Casas Grandes No 69. México, D F. 296 p.

Brousse, G y Loussert, R. 1980. El olivo. Ed. MundiPrensa. España. 533 p.

Castillo, A. 1983. Enraizamiento de Cedrelinga catenaeformis Ducke, aplicando Seradix-3 y bajo diferentes condiciones ambientales en Pichanaki (Chanchamayo, Junín), Tesis Ing. Forestal UNCP, Huancayo, Perú.

Claussi, A. 1986. Propagación vegetativa del tornillo mediante enraizamiento de estacas en la zona de Jenaro Herrera, Iquitos (documento preliminar).

Claussi, A.; Marmillod, D.; Blaser, J. 1992. Descripción silvicultural de las plantaciones forestales de Jenaro Herrera. Instituto de Investigaciones de la Amazonía Peruana, Centro de Investigaciones de J.H, Iquitos, Perú. 334.

Davison, J. 1974. Reproduction of Eucalyptus deglupta by cuttings. Journal of Forestry Science. New Zeland. 4(2): 191-203

Díaz, E; Salazar, R; Mesén, F. 1991. Enraizamiento de estacas juveniles de Gmelina arborea Linn. Silvoenergía. 49:1-4.

Gonçalez, J.; Gonçalvez, M. 2001. Valorização de duas espécies de madeira Cedrelinga catenaeformis e Enterolobium shomburgkii para a indústria madeireira. Brasil Florestal. $\mathrm{N}^{\circ} 70$.

Flores, Y. 2004. Guía para el reconocimiento de regeneración natural de especies forestales de la región Ucayali. INIA. EEP. Pucallpa, Perú. 80 p.

Flores, Y. 2010. Crecimiento y productividad de plantaciones forestales en la amazonía peruana. INIA. Lima. $60 \mathrm{p}$.

Hartmann, T.; Kester, D. 1972. Propagación de plantas: principios y prácticas. Trad. Por Marino Ambrosio. La Habana. Instituto Cubano del Libro. $693 \mathrm{p}$.

Hartmann, T.; Kester, D. 1998. Propagación de plantas. Principios y práctica. Editorial Continental. México. $757 \mathrm{p}$.

Hartmann, T; Kester, D and Davies, T. 1992. Plant propagation. Principles and Practices. Fifth.

International Union For Conservation of Nature and Natural Resourses (IUCN). Publicado en internet. http://www.iucnredlist.org (Accesado 2007).

Leakey, B. 1983. Stockplant factors affecting root initiation in cuttings of Triplochiton scleroxylon $\mathrm{K}$. Schum., and Indigenous Hardwood of West Africa. Journal of Horticultural science. 58(2):277-290.

Leakey, B. 1985. The capacity for vegetative propagation in trees. In: Canell, MGR; Jackson, JE (eds). Trees as corp plants. Midlothian, UK, Institute of Terrestrial Ecology. 110-133 pp. 
Leakey, B. 1987. Clonal Forestry in the tropics-A review of developments, strategies and opportunities. Commonwealth Forestry Review. Inglaterra. 66: 61-75.

Leakey, B.; Mesén, F.; Tchoundjeu, Z.; Longman, A.; Dick, J.; Newton, A.; Martin, A.; Grace, J.; Munro, C.; Muthoka, N. 1990. Low technology techniques for vegetative propagation of tropical tress. En: Commonwealth Forestry Rewiew. 66 (1): 61-75.

Leakey, B y Mesén, F. 1991. Métodos de propagación vegetativa en árboles tropicales: enraizamiento de estacas suculentas. Capítulo 10. In Manual sobre mejoramiento genético forestal con referencia especial a América Central. Cornelius JP, Mesen F, Corea E (eds.), Proyecto Mejoramiento Genético Forestal, CATIE, Turrialba, Costa Rica. pp. 135153

Levi, D. 1987. Propagación por estacas del tornillo (Cedrelinga catenaeformis Ducke), con aplicación de estimulante del enraizamiento bajo condiciones de Tingo María. Universidad Nacional Agraria de la selva. Tesis para Obtener el Título Profesional de Ingeniero Recursos Naturales. Tingo María. 97p.

Loayza, M. 2004. El cambio desde adentro. OIMT A ctualidad Forestal Tropical. Vol.12.N ${ }^{\circ}$ 2.Publicadoeninternet.http//itto.or.jp/liv e/Live_Servet/728/tfu.2004.02(03-06) PDF.

Longman, A. 1993. Árboles tropicales: manuales de propagación. Enraizamiento de estacas de árboles tropicales. London, United Kingdom: Commonwealth Science Concil. $131 \mathrm{p}$.

Maruyama, E. 1986. Pruebas de enraizamiento de estacas de tornillo Cedrelinga catenaeformis Ducke. EE.A. Von Humboldt. Pucallpa. Perú.

Maruyama, E. 1987. Respuesta de tornillo (C. catenaeformis) al Almacenamiento de las Semillas y Comportamiento de la Regeneración Natural en la zona de Alexander von Humboldt. Tesis de Ing. Forestal. Pucallpa, Perú. 178p.

Mateo, S; Vargas, H; López, P; Jasso, M. 2000. Enraizado de estacas juveniles de cinco especies de coníferas ornamentales. Ciencia For. México. 23:29-39.

Mesén, F. 1992. Hacia el desarrollo de técnica de silvicultura clonal para el pequeño finquero. Chasqui. Turrialba, Costa Rica. 28: 6-18

Mesén, F. 1993. Vegetative Propagation of Central American Hardwoods. Thesis Ph.D. Edinburgh, Scotland, University of Edinburgh. Institute of Terrestrial Ecology. 231 p.

Mesén, F. 1998. Enraizamiento de estacas juveniles de especies forestales: uso de propagadores de subirrigación. Serie Técnica, Manual Técnico Nº 30.

\section{CATIE. Turrialba, Costa Rica. $36 \mathrm{p}$}

Mesén, F; Leakey, B; Newton, A. 1992. Hacia el desarrollo de técnicas de silvicultura clonal para el pequeño finquero. Boletín informativo sobre recursos naturales renovables. El Chasqui. CATIE. Turrialba, Costa Rica. 28: 6-18 p.

Mesén, F y Trejos, E. 1997. Propagación vegetativa de San Juan (Vochysia guatemalensis Donn. Smith) mediante enraizamiento de estacas juveniles. Revista Forestal Centroamericana. 21:19-24.

Mesén, F; Leakey, B; Newton, A. 1996. Propagadores de subirrigación: un sistema simple y económico para la propagación de estaquillas forestales. In Avances en la Producción de Semillas Forestales en América Latina. Memorias. (Ed. Salazar, R.). Managua, Nicaragua, 1995. pp. 101-110.

Moe, R and Andersen, A. 1988. Stockplant environment and subsequent adventitious rooting. In Adventitious Root Formation in Cuttings. (Eds. Davis, T.D., Haissig, B.E. and Sankhla, N.) B.E. Dioscorides Press, Portland, Oregon. 214-234 pp.

Nuñez, Y. 1997. Propagación vegetativa del cristóbal (Platymiscium pinnatum, Benth); pilón (Hyeronima alchorneoides, Allemo) y surá (Terminalia oblonga, Ruiz \& Pavón) mediante el enraizamiento de estacas juveniles. Tesis Mg. Sc. CATIE. Turrialba, Costa Rica. $172 \mathrm{p}$

Ponce-Alvarez, S. 2005. Investigation on some physical and mechanical properties of Cedrelinga cateniformis (Ducke) Ducke from a 31 year-old forest plantation grown at the Centro de Investigación Jenaro Herrera (CIJH) in the Peruvian Amazon. Georg-August-University. Thesis to obtain the degree of Master of Science in Tropical and International Forestry. Germany. 71p.

Protected Areas Programme. 2001. Parks: Integrated conservation and Development.Publict. internethttp://www.iucn.org/themes/wcpa/pubs/p dfs/PARKS/park_ju.

Quijada, R. 1980. Métodos de propagación vegetativa. En mejora genética de árboles forestales. FAO. DANIDA. Sirve. Montroso. Roma, Italia. 341 p.

Ruiz, G; Vargas, H; Cetina, A; Villegas, M. 2005. Efecto del ácido indolbutírico (AIB) y tipo de estaca en el enraizado de Gmelina arborea Roxb. Rev. Fitotec. Mex. Vol. 28 (4), pp. 319-326.

Ruiz, H. 2009. Efecto de cuatro dosis de ácido indolbutírico y tres tipos de estacas en el enraizamiento de Sacha inchi (Plukenetia volubilis L.) en San Martín. Tesis para optar el título de ingeniero Agrónomo. Facultad de Agronomía de la Universidad Nacional de la Selva. 123 P.

Schwyzer, A. 1981. El tornillo (Cedrelinga 
catenaeformis) proyecto de asentamiento rural integral Jenaro Herrera, Boletín técnico 15, Iquitos, Perú.

Soudre, M.; Portal, E. 2007. Propagación vegetativa de estaquillas de tornillo (Cedrelinga cateniformis Ducke) en cámaras de subirrigación. Informe técnico. Subproyecto silvicultura de bolaina en plantaciones y sucesiones secundarias en Ucayali. Programa de Ecosistemas Terrestes (PET). IIAP. Ucayali, Perú. 8p

Soudre, M.; Mesén, F.; Del Castillo, D.; Guerra, H. 2008. Memoria del curso internacional: Bases Técnicas Para la Propagación Vegetativa de Árboles Tropicales Mediante Enraizamiento de Estaquillas. Proyecto PROVEFOR. Pucallpa, Perú. $104 \mathrm{p}$

Tello, F.; Quevedo, A.; Gasche. 2004. Sistema de incentivos para el manejo de bosques de Loreto: El caso de los recursos forestales maderables. Informe Final. Instituto de investigación de la Amazonía Peruana. $170 \mathrm{p}$.

Universidad Nacional de Ucayali. Boletín de la Estación Meteorológica Principal UNU. C. Federico Basadre $\mathrm{Km}$ 6. Pucallpa. Ucayali. Promedios 2009 al 2011

Valderrama, F. 2003. Plantas de .importancia económica y ecológica en el jardín botánicoArboretum El Huayo, Iquitos. Folia Amazónica 14 (1): $159-175$

Wright, W. 1964. Mejoramiento genético de los árboles forestales. FAO. Estudios de Silvicultura y Productos Forestales. 16: 436 p

Zobel, B.; Talber, J. 1988. Técnicas de mejoramiento genético de árboles forestales. México. Ed. Limusa. $554 \mathrm{p}$. 